\title{
Meis1 regulates postnatal cardiomyocyte cell cycle arrest
}

\author{
Ahmed I. Mahmoud ${ }^{\# 1}$, Fatih Kocabas ${ }^{\# 1}$, Shalini A. Muralidhar ${ }^{\# 1}$, Wataru Kimura ${ }^{1}$, Ahmed S. \\ Koura $^{2}$, Suwannee Thet ${ }^{1}$, Enzo R. Porrello ${ }^{3}$, and Hesham A. Sadek ${ }^{1}$ \\ ${ }^{1}$ Department of Internal Medicine, Division of Cardiology, The University of Texas Southwestern \\ Medical Center, Dallas, Texas 75390, USA \\ ${ }^{2}$ School of Medicine, Ain Shams University, Cairo, 1156, Egypt \\ ${ }^{3}$ School of Biomedical Sciences, The University of Queensland, St Lucia, Queensland 4072, \\ Australia. \\ \# These authors contributed equally to this work.
}

\section{Abstract}

The neonatal mammalian heart is capable of substantial regeneration following injury through cardiomyocyte proliferation ${ }^{1,2}$. However, this regenerative capacity is lost by postnatal day 7 and the mechanisms of cardiomyocyte cell cycle arrest remain unclear. The homeodomain transcription factor Meis 1 is required for normal cardiac development but its role in cardiomyocytes is unknown ${ }^{3,4}$. Here we identify Meis1 as a critical regulator of the cardiomyocyte cell cycle. Meis 1 deletion in mouse cardiomyocytes was sufficient for extension of the postnatal proliferative window of cardiomyocytes, and for re-activation of cardiomyocyte mitosis in the adult heart with no deleterious effect on cardiac function. In contrast, overexpression of Meis1 in cardiomyocytes decreased neonatal myocyte proliferation and inhibited neonatal heart regeneration. Finally, we show that Meis1 is required for transcriptional activation of the synergistic CDK inhibitors p15, p16 and p21. These results identify Meis1 as a critical transcriptional regulator of cardiomyocyte proliferation and a potential therapeutic target for heart regeneration.

\begin{abstract}
The hallmark of heart failure is the progressive nature of the disease, and the inability of the adult heart to regenerate after injury. Nonetheless, limited myocyte turnover does in fact occur in the adult mammalian heart; however, this is insufficient to restore contractile function following injury ${ }^{5,6}$. In contrast, the neonatal heart has a proliferative capacity reminiscent of lower vertebrates ${ }^{1,2,7}$. These findings indicate that the key to unlocking the regenerative potential of the adult mammalian heart may lie within the developmental transitions occurring during neonatal life. Given that cardiac regeneration in neonatal mice,
\end{abstract}

C2013 Macmillan Publishers Limited. All rights reserved

Correspondence and requests for materials should be addressed to H.A.S. (hesham.sadek@utsouthwestern.edu).

Supplementary Information is available in the online version of the paper.

Author Contributions A.I.M, F.K., S.A.M., E.R.P. and H.A.S. designed the experiments. A.I.M., F.K., S.A.M., W.K. and S.T. performed the experiments. A.I.M., F.K., S.A.M., A.S.K., E.R.P. and H.A.S. analysed the data. A.I.M., F.K., S.A.M. and H.A.S. made the figures. A.I.M. and H.A.S. wrote the manuscript. All authors approved the manuscript.

The authors declare no competing financial interests. 
as well as in adult zebrafish ${ }^{1,2,8,9}$, is primarily mediated by cardiomyocyte proliferation, it is crucial to identify the molecular mechanisms responsible for postnatal cardiac cell cycle arrest.

The transcriptional networks that govern embryonic heart development have been extensively studied. In contrast, little is known about transcriptional regulation of postnatal cardiomyocyte cell cycle arrest. Recent evidence suggests that Meis transcription factors, which belong to the TALE (three amino acid loop extension) family of homeodomain transcription factors, are central regulators of cardiac differentiation during embryonic development ${ }^{3,4}$. While global deletion shows that Meis 1 is required for normal embryonic haematopoiesis and heart development ${ }^{10-12}$, the role of Meis1 in the postnatal heart remains unclear. We recently demonstrated that Meis1 is an important regulator of haematopoietic stem cells (HSCs) quiescence ${ }^{13}$. Moreover, our initial screens identified Meis1 as a potential transcriptional regulator of neonatal heart regeneration. Therefore, we conducted this study to determine the role of Meis1 in regulation of cardiomyocyte cell cycle. We first examined the expression pattern of Meis1 during neonatal heart development and regeneration.

Quantitative PCR with reverse transcription (qRT-PCR) showed a modest increase in Meis 1 expression between postnatal day 1 (P1) and P7 (Fig. 1a). Moreover, immunostaining revealed that Meis1 is expressed in the heart as early as P4, and throughout adulthood (Supplementary Fig. 1a). Meis1 was localized to perinuclear regions in neonatal cardiomyocytes at P1, but became nuclear localized by P4-P7 (Fig. 1c and Supplementary Fig. 1b). Myocardial infarction (MI) at P1, which is associated with an induction of robust cardiomyocyte proliferation at day 7 post-MI ${ }^{2}$, was associated with a modest decrease in the expression of Meis 1 , whereas Meis 1 mRNA expression levels were significantly increased following MI at P7, a time point coinciding with lack of mitotic induction of cardiomyocytes (Fig. 1b).

We also examined the expression of Meis 1 isoforms in the heart postnatally, and we found that isoform Meis $1 B$ is the predominant isoform (Supplementary Fig. 1c, d). Finally, given that Meis 1 transcriptional activity is known to be tightly linked to other Hox genes, we profiled the entire Hox family by qRT-PCR (Supplementary Fig. 1e). Intriguingly, we found that the three most upregulated genes were Hoxa10, Hoxa11, and Hoxd12 (all belonging to the AbdB-like paralogue of Hox genes), which along with Hoxa9, and Hoxb13 are the only Hox proteins (out of 37 known mammalian Hox genes) known to interact with Meis $1{ }^{14}$. These Hox proteins stabilize Meis1 DNA binding and enhance its transcriptional activity.

To elucidate a potential role for Meis 1 in cardiomyocyte proliferation, we performed in vitro siRNA knockdown using rat neonatal cardiomyocytes, and found that Meis 1 knockdown (Fig. 1d) resulted in a threefold increase in cardiomyocyte proliferation (Fig. 1e). Next, we generated cardiomyocyte-specific Meisl knockout (KO) mice by crossing Meis $1^{\mathrm{f} / \mathrm{f}}$ mice with aMHC-Cre mice (Fig. 2a). qRT-PCR (Fig. 2b) using isolated cardiomyocytes from Meis $1^{\mathrm{f} / \mathrm{f}}$ aMHC-Cre (Meisl KO) compared to Meis ${ }^{+/+}$aMHC-Cre (control) mouse hearts confirmed a change in gene expression consistent with Meis 1 deletion in cardiomyocytes. Phenotypic characterization of Meisl KO mice at P14 (1 week beyond the normal window of postnatal cardiomyocyte cell cycle arrest) demonstrated that heart size (Fig. 2c, d) and cardiac function (Fig. 2e) were unaffected by Meis 1 deletion. However, Meisl KO 
cardiomyocytes were smaller compared to control cardiomyocytes (Fig. 2f), which may imply that the cardiomyocyte number is increased in Meis $1 \mathrm{KO}$ (smaller cardiomyocyte size, with no change in heart to body weight ratio). Therefore, we examined the Meis 1 KO hearts for myocyte proliferation using the mitosis marker $\mathrm{pH} 3$ (phosphorylated histone $\mathrm{H} 3$ ) and the cytokinesis marker Aurora B kinase. Meis 1 deletion resulted in induction of cardiomyocyte proliferation as quantified by an increase in the number of $\mathrm{pH} 3^{+} \mathrm{TnnT}^{+}$(troponin $\mathrm{T} 2$ ) cells (>9 fold) (Fig. 2g, top row of images and bar graph). Moreover, we show that sarcomere disassembly, a hallmark for cardiomyocyte proliferation, was evident in the Meisl KO heart sections (Fig. 2g, bottom left image), whereas neighbouring myocardium had normal sarcomeric structure (Fig. 2g, bottom right image). In addition, we found that the cytokinesis marker Aurora B kinase was markedly expressed in the cleavage furrow between proliferating myocytes in the KO hearts ( $>5$ fold) (Fig. $2 \mathrm{~h}$ ). We also found a significant increase in the number of 5-bromodeoxyuridine (BrdU) positive cardiomyocytes in the Meis1 KO hearts (Fig. 2i).

To determine whether deletion of Meisl increases the total number of cardiomyocytes, we isolated adult cardiomyocytes from wild-type and $\mathrm{KO}$ hearts by collagenase digestion and found a significant increase in the total number of cardiomyocytes in Meis 1 KO hearts (Fig. $2 \mathrm{j}$, left graph). This was accompanied by an increase in the percentage of mononucleated cardiomyocytes, and a decrease in the percentage of binucleated cardiomyocytes (Fig. $2 \mathrm{j}$, right graph), possibly indicative of proliferation of a mononuclear myocyte population ${ }^{15}$, or reflecting the nucleation status of mitotic cardiomyocytes ${ }^{16,17}$. These results demonstrate that Meis 1 deletion affects both cell cycle activity and nucleation of cardiomyocytes postnatally. Finally, Meis 1 deletion did not result in an increase in myocyte apoptosis by TdT-mediated dUTP nick end labelling (TUNEL) staining (Fig. 2k).

Next, we examined the effect of Meis 1 deletion on the adult heart. Heart size (Supplementary Fig. 2a) and cardiac function were normal in adult mice at the postnatal stages of 28 days and 7 months (Supplementary Fig. 2b, c). There was no difference in the size of cardiomyocytes in the adult heart following Meisl deletion in late gestation (Supplementary Fig. 2d). Similarly, we examined cardiomyocyte mitosis in adult hearts and found that Meisl KO hearts continued to show increased mitosis indices even in adulthood, although cardiomyocyte proliferation appeared to decrease with age (Supplementary Fig. 2e, f), with no increase in cardiomyocyte apoptosis (Supplementary Fig. 2g). In addition, there was no change in the expression pattern of hypertrophy markers except for a modest reduction in ANP (also known as natriuretic peptide type A) (Supplementary Fig. 2h).

Although sustained induction of cardiomyocyte cell cycle from birth by overexpression of cell cycle regulators has been previously achieved ${ }^{16,17}$, cell cycle re-entry in adult cardiomyocytes appears to be limited to a subpopulation of mononucleated myocytes in one report ${ }^{15}$, and another study suggested that cell cycle re-entry is associated with contractile dysfunction ${ }^{18}$. Therefore, we examined whether conditional deletion of Meis 1 in the adult heart could induce cardiomyocyte cell cycle re-entry. We crossed Meis $1^{\mathrm{f} / \mathrm{f}}$ mice with aMHC-MerCreMer mice, which allowed for specific deletion of Meis 1 in cardiomyocytes following tamoxifen administration, these inducible Meisl knockout mice are hereafter referred to as Meis 1 iKO. We then confirmed Meisl deletion in the majority of 
cardiomyocytes (Fig. 3a, b), and we found that Meis 1 deletion did not affect cardiac morphology or fibrosis (Fig. 3c); however, the heart to body weight ratio was increased 6 weeks after Meis 1 deletion (Fig. 3d). Further characterization of the Meis1 iKO mice demonstrated normal cardiac function (Fig. 3e) and cardiomyocyte size (Fig. 3f). Intriguingly, the number of mitotic cardiomyocytes, detected by $\mathrm{pH} 3$ staining, increased by more than tenfold (Fig. 3g), with a significant increase in cytokinesis (Fig. 3h) 1 week after tamoxifen administration. In addition, we found that the Meis 1 iKO hearts had increased number of cardiomyocytes (Fig. 3i, left), and a higher number of mononucleated cardiomyocytes (Fig. 3i, right). Finally, we did not detect any significant differences in apoptosis in the Meisl iKO hearts (Fig. 3j). Collectively, these results demonstrate that deletion of Meis 1 in adult cardiomyocytes is sufficient for induction of cell cycle re-entry.

To determine whether forced Meisl expression can inhibit neonatal cardiomyocyte proliferation, we generated a cardiac-specific Meisl overexpressing mouse (Meisl OE) by crossing pTRE-Meis1 mice with aMHC-tTA mice to allow for specific overexpression of Meis1 in cardiomyocytes (Fig. 4a) around birth in the absence of tetracyclin. We used a Meis1 line that overexpressed Meis1 by approximately 2.5-fold (Fig. 4b). Overexpression of Meis 1 did not result in a significant increase in heart to body weight ratio (Fig. 4c, d), normal systolic function (Fig. 4e) and increased cardiomyocyte size (Fig. 4f). This was associated with a modest increase in the hypertrophy marker ANP (Fig. 4g). Despite the increase in cardiomyocyte size in Meis 1 (OE) hearts, the lack of decrease in heart to body weight ratio most probably reflects a decrease in the number of cardiomyocytes. This is supported by a decrease in the number of mitotic cardiomyocytes in the neonatal Meis 1 (OE) hearts (Fig. 4h). More notably, Meisl overexpression inhibited neonatal heart regeneration following induction of MI at P1 (Fig. 4i-k), whereas the wild-type hearts regenerated normally. Finally, Meisl overexpression in cardiomyocytes resulted in upregulation of CDK inhibitors, most significantly p21 (also known as Cdkn1a) (Fig. 4l). These results indicate that Meisl overexpression in the neonatal heart results in premature cardiomyocyte cell cycle arrest.

In order to determine the mechanism by which Meis1 regulates cardiomyocyte proliferation, we performed a cell cycle PCR array. We found that Meis 1 deletion resulted in downregulation of cyclin dependent kinase inhibitors in isolated cardiomyocytes, including members of the Ink4b-Arf-Ink4a locus (p16, p15 and p19 ${ }^{\mathrm{ARF}}$ ) and CIP/KIP family (p21 and p57), as well as upregulation of a number of positive regulators of the cell cycle (Supplementary Fig. 3a, b). qRT-PCR confirmed these results (Supplementary Fig. 4a). Of all the dysregulated cell cycle genes, we identified conserved Meis1 consensus binding sequences in the promoter region of only two loci, namely the Ink4b-Arf-Ink4a (which includes $\mathrm{p} 16^{\mathrm{INK} 4 \mathrm{a}}, \mathrm{p} 19^{\mathrm{ARF}}$ and $\mathrm{p} 15^{\mathrm{INK} 4 \mathrm{~b}}$ ) (Supplementary Fig. 4b, left panel) and $p 21$ loci (Supplementary Fig. 4c, left panel) using the UCSC genome browser (http:// genome.ucsc.edu).

To test if Meis1 can transcriptionally activate Ink4b-Arf-Ink4a and $p 21$, we generated luciferase reporter constructs containing the conserved Meis1 binding motifs. Luciferase reporter assays with INK4b-ARF-INK4a-pGL2 (Supplementary Fig. 4b, right panel) and p21-pGL2 reporters (Supplementary Fig. 4c, right panel) demonstrated a dosedependent 
activation by Meis1. Mutation of the putative Meis1 binding sites abolished the Meis1dependent activation of the luciferase reporters (Supplementary Fig. 4b, c). Finally, we demonstrated an in vivo interaction between Meis1 and Ink4b-Arf-Ink4a and $p 21$ promoters in the adult mouse heart by chromatin immunoprecipitation (Supplementary Fig. 4d).

Several studies over the past two decades have implicated cell cycle regulators in cardiomyocyte cell cycle arrest. Cardiomyocyte cell cycle exit is associated with downregulation of positive cell cycle regulators (CDK2, CDK3, CDK4, CCND1 and CDK cofactors) and induction of cell cycle inhibitors (p21, TSC2, p130 and Rb) ${ }^{19-22}$. Consistently, cardiomyocyte specific overexpression of Cyclin D2, which is a CDK cofactor that positively regulates cell cycle progression, or deletion of $\mathrm{p} 130$ and $\mathrm{Rb}^{18}$, leads to cardiomyocyte cell cycle entry ${ }^{16}$. In addition, deletion of CDKI p2 $7^{\mathrm{Kip} 1}$ or immunodepletion of $\mathrm{p} 21^{\mathrm{Cip} 1}$ in cardiomyocytes is associated with a trend towards progression to $\mathrm{S}$-phase ${ }^{21}$. However, the mechanism of postnatal regulation of these direct cell cycle effector molecules in cardiomyocytes has been unclear. The current study identifies Meis1 as a critical transcriptional regulator of cardiomyocyte cell cycle, upstream of two synergistic CDKI inhibitors. Our results also demonstrate that Meis 1 deletion results in upregulation of a number of positive cell cycle regulators such as MCM3, Chek1 and Ccnd2, and downregulation of other negative regulators of the cell cycle such as APbb1, TP53 and Gpr132 and others, all which may play a role in the cell cycle effect of Meis 1 deletion (Supplementary Fig. 3). Although the mechanism of activation of Meis1 in the postnatal heart is not quite fully understood, our results demonstrate that Meis1 expression coincides with upregulation of 3 of the only 5 Hox genes that are known to interact with Meis1, stabilize its DNA binding and enhance its transcriptional activity. Therefore, it would be important for future studies to define the transcriptional network involved in mediating the effect of Meis1 on postnatal cardiomyocytes.

The diverse functions of Meis1 in different organs have begun to emerge. We recently identified Meis 1 as an important regulator of anaerobic glycolysis in the HSC hypoxic niche through regulation of Hif-1a and Hif-2a, in which conditional Meis 1 deletion resulted in a metabolic switch from glycolysis to oxidative phosphorylation resulting in apoptosis of $\mathrm{HSCs}^{23}$. In contrast, Meis 1 deletion in cardiomyocytes is not associated with increased apoptosis, probably owing to the dependence of cardiomyocytes on oxidative metabolism compared to the glycolytic HSCs.

Although it was previously thought that the heart is a post-mitotic organ, it is now clear that measurable myocyte turnover can occur in the adult mouse ${ }^{6,24}$ and human ${ }^{25,26}$ hearts. Recent reports indicate that cardiomyocyte proliferation is the main source of myocyte turnover in the adult heart ${ }^{27}$, and can even mediate adult heart regeneration ${ }^{28}$. Our results indicate that reversal of adult cardiomyocyte cell cycle arrest may be conceivable through careful analysis of early postnatal events, and identifies Meis1 as a key regulator of postnatal cardiomyocyte proliferation. 


\section{METHODS SUMMARY}

Neonatal mice were subjected to myocardial infarction surgeries as previously described ${ }^{2}$. Experimental animal protocols were approved by the Institutional Animal Care and Use Committee of the University of Texas Southwestern Medical Center. All experiments were performed on age and sex matched mice, with an equal ratio of male to female mice. Results are expressed as mean \pm s.e.m. An unpaired Student's $t$ test was used to determine statistical significance of all samples. $* P<0.05$, $* * P<0.01$ were considered statistically significant.

\section{Supplementary Material}

Refer to Web version on PubMed Central for supplementary material.

\section{Acknowledgments}

We thank J. Shelton for help with histology, J. Cabrera for help with figures, N. Copeland and N. Jenkins for providing the Meis 1 KO mice, S. Das for statistics consultation, K. Luby-Phelps for help with microscopy, A. Bugde for confocal assistance, D. Farrar for discussions, H. Nguyen for help with echocardiography, as well as K.Sheth, A. Mercadel and Z. Sadek for technicalassistance. This work is supported by grants from the American Heart Association (Grant in Aid) (H.A.S.), the Gilead Research Scholars Program in Cardiovascular Disease (H.A.S.), the Foundation for Heart Failure Research, NY, and the National Institutes of Health (1R01HL115275-01) (H.A.S.).

\section{References}

1. Porrello ER, et al. Transient regenerative potential of the neonatal mouse heart. Science. 2011; 331:1078-1080. [PubMed: 21350179]

2. Porrello ER, et al. Regulation of neonatal and adult mammalian heart regeneration by the miR-15 family. Proc. Natl Acad.Sci. USA. 2013; 110:187-192. [PubMed: 23248315]

3. Paige SL, et al. A temporal chromatin signature in human embryonic stem cells identifies regulators of cardiac development. Cell. 2012; 151:221-232. [PubMed: 22981225]

4. Wamstad JA, et al. Dynamic and coordinated epigenetic regulation of developmental transitions in the cardiac lineage. Cell. 2012; 151:206-220. [PubMed: 22981692]

5. Bergmann O, et al. Evidence for cardiomyocyte renewal in humans. Science. 2009; 324:98-102. [PubMed: 19342590]

6. Hsieh PC, et al. Evidence from a genetic fate-mapping study that stem cells refresh adult mammalian cardiomyocytes after injury. Nature Med. 2007; 13:970-974. [PubMed: 17660827]

7. Heallen T, et al. Hippo pathway inhibits Wnt signaling to restrain cardiomyocyte proliferation and heart size. Science. 2011; 332:458-461. [PubMed: 21512031]

8. Kikuchi K, et al. Primary contribution to zebrafish heart regeneration by gata $4^{+}$cardiomyocytes. Nature. 2010; 464:601-605. [PubMed: 20336144]

9. Jopling C, et al. Zebrafish heart regeneration occurs by cardiomyocyte dedifferentiation and proliferation. Nature. 2010; 464:606-609. [PubMed: 20336145]

10. Azcoitia V, Aracil M, Martinez AC, Torres M. The homeodomain protein Meis1 is essential for definitive hematopoiesis and vascular patterning in the mouse embryo. Dev Biol. 2005; 280:307320. [PubMed: 15882575]

11. Hisa T, et al. Hematopoietic, angiogenic and eye defects in Meis1 mutant animals. EMBO J. 2004; 23:450-459. [PubMed: 14713950]

12. Imamura $\mathrm{T}$, et al. Frequent co-expression of HoxA9 andMeis 1 genesininfant acute lymphoblastic leukaemia with $M L L$ rearrangement. Br. J. Haematol. 2002; 119:119-121. [PubMed: 12358913]

13. Kocabas F, et al. Meis1 regulates the metabolic phenotype and oxidant defense of hematopoietic stem cells. Blood. 2012; 120:4963-4972. [PubMed: 22995899] 
14. Shen WF, et al. AbdB-like Hox proteins stabilize DNA binding by the Meis1 homeodomain proteins. Mol. Cell. Biol. 1997; 17:6448-6458. [PubMed: 9343407]

15. Bersell K, Arab S, Haring B, Kuhn B. Neuregulin1/ErbB4 signaling induces cardiomyocyte proliferation and repair of heart injury. Cell. 2009; 138:257-270. [PubMed: 19632177]

16. Pasumarthi KB, Nakajima H, Nakajima HO, Soonpaa MH, Field LJ. Targeted expression of cyclin D2 results in cardiomyocyte DNA synthesis and infarct regression in transgenic mice. Circ. Res. 2005; 96:110-118. [PubMed: 15576649]

17. Gude N, et al. Akt promotes increased cardiomyocyte cycling and expansion of the cardiac progenitor cell population. Circ. Res. 2006; 99:381-388. [PubMed: 16840722]

18. Sdek $\mathrm{P}$, et al. $\mathrm{Rb}$ and $\mathrm{p} 130$ control cell cycle gene silencing to maintain the postmitotic phenotype in cardiac myocytes. J. Cell Biol. 2011; 194:407-423. [PubMed: 21825075]

19. Pasumarthi KB, Field LJ. Cardiomyocyte cell cycle regulation. Circ. Res. 2002; 90:1044-1054. [PubMed: 12039793]

20. Walsh S, Ponten A, Fleischmann BK, Jovinge S. Cardiomyocyte cell cycle control and growth estimation in vivo-an analysis based on cardiomyocyte nuclei. Cardiovasc. Res. 2010; 86:365373. [PubMed: 20071355]

21. Poolman RA, Gilchrist R, Brooks G. Cell cycle profiles and expressions of p21 CIP1 AND P27KIP1 during myocyte development. Int. J. Cardiol. 1998; 67:133-142. [PubMed: 9891946]

22. MacLellan WR, et al. Overlapping roles of pocket proteins in the myocardium are unmasked by germ line deletion of p130 plus heart-specific deletion of Rb. Mol. Cell. Biol. 2005; 25:24862497. [PubMed: 15743840]

23. Simsek T, et al. The distinct metabolic profile of hematopoietic stem cells reflects their location in a hypoxic niche. Cell Stem Cell. 2010; 7:380-390. [PubMed: 20804973]

24. Smart N, et al. De novo cardiomyocytes from within the activated adult heart after injury. Nature. 2011; 474:640-644. [PubMed: 21654746]

25. Beltrami AP, et al. Evidence that human cardiac myocytes divide after myocardial infarction. N. Engl. J. Med. 2001; 344:1750-1757. [PubMed: 11396441]

26. Mollova M, et al. Cardiomyocyte proliferation contributes to heart growth in young humans. Proc. Natl Acad. Sci. USA. 2013; 110:1446-1451. [PubMed: 23302686]

27. Senyo SE, et al. Mammalian heart renewal by pre-existing cardiomyocytes. Nature. 2013; 493:433-436. [PubMed: 23222518]

28. Eulalio A, et al. Functional screening identifies miRNAs inducing cardiac regeneration. Nature. 2012; 492:376-381. [PubMed: 23222520] 

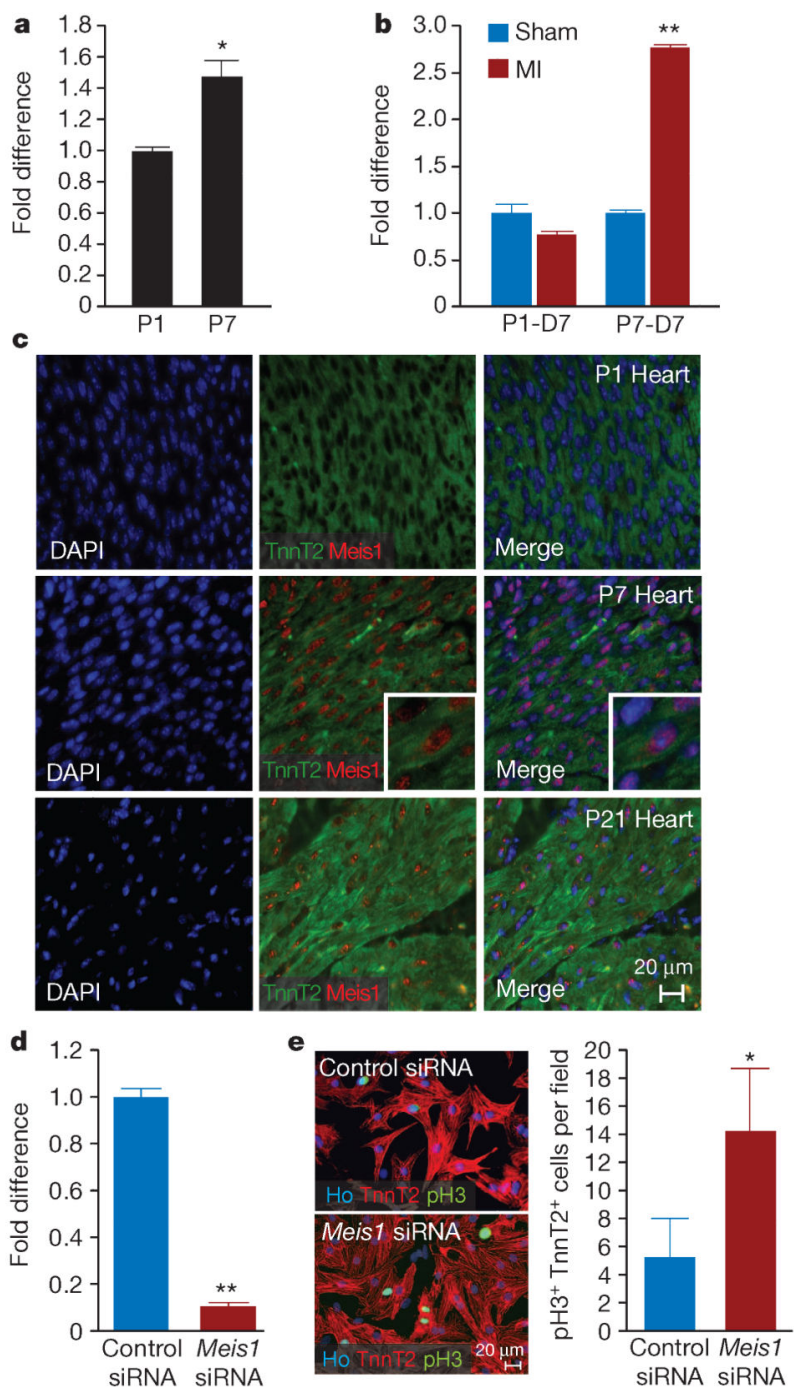

Figure 1. Expression profile of Meis1 in the heart

a, qRT-PCR showing increased expression of Meis1 at postnatal day 7 (P7), a time point that coincides with cell cycle arrest of cardiomyocytes. b, qRT-PCR showing expression levels of Meis 1 following Sham or MI at P1 or P7. Samples were collected 7 days after the injury was performed (D7). c, Expression profile of Meis1 in cardiomyocytes. Top row, Meis1 expression is absent at P1. Middle and bottom rows, nuclear localization of Meis1 in cardiomyocytes at $\mathrm{P} 7$ and P21, respectively. d, qRT-PCR showing knockdown of Meis 1 in rat neonatal cardiomyocytes using Meis 1 siRNA. e, Cardiomyocyte mitosis following Meis 1 knockdown. Left panel, immunostaining showing co-localization of pH3, TnnT2 and Hoechst $(\mathrm{Ho})$ in rat neonatal cardiomyocytes. Right panel, quantification of the $\mathrm{pH} 3^{+}$ $\mathrm{TnnT}^{+}$nuclei in control and Meis 1 siRNA treated cardiomyocytes. Quantitative analysis represents counting of multiple fields from three independent samples per group; ${ }^{*} P<0.05$, $* * P<0.01$; error bars represent mean \pm s.e.m. 


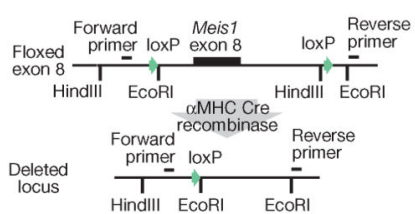

b

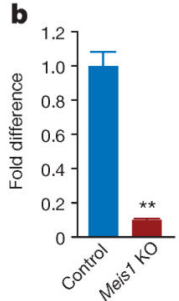

$\mathbf{g}$

f
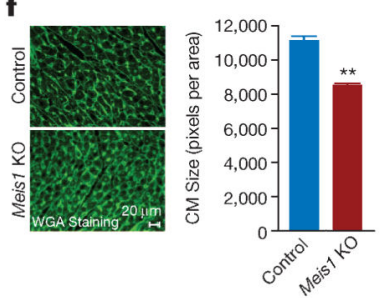

c

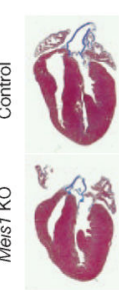

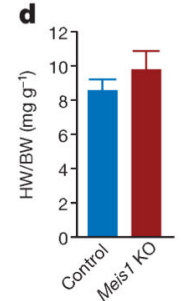

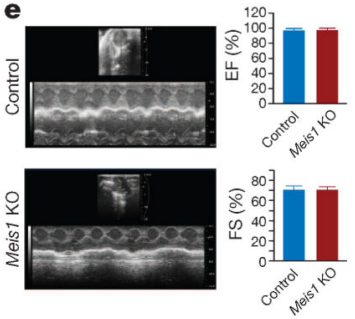

h

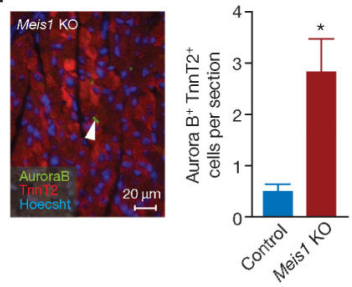

i
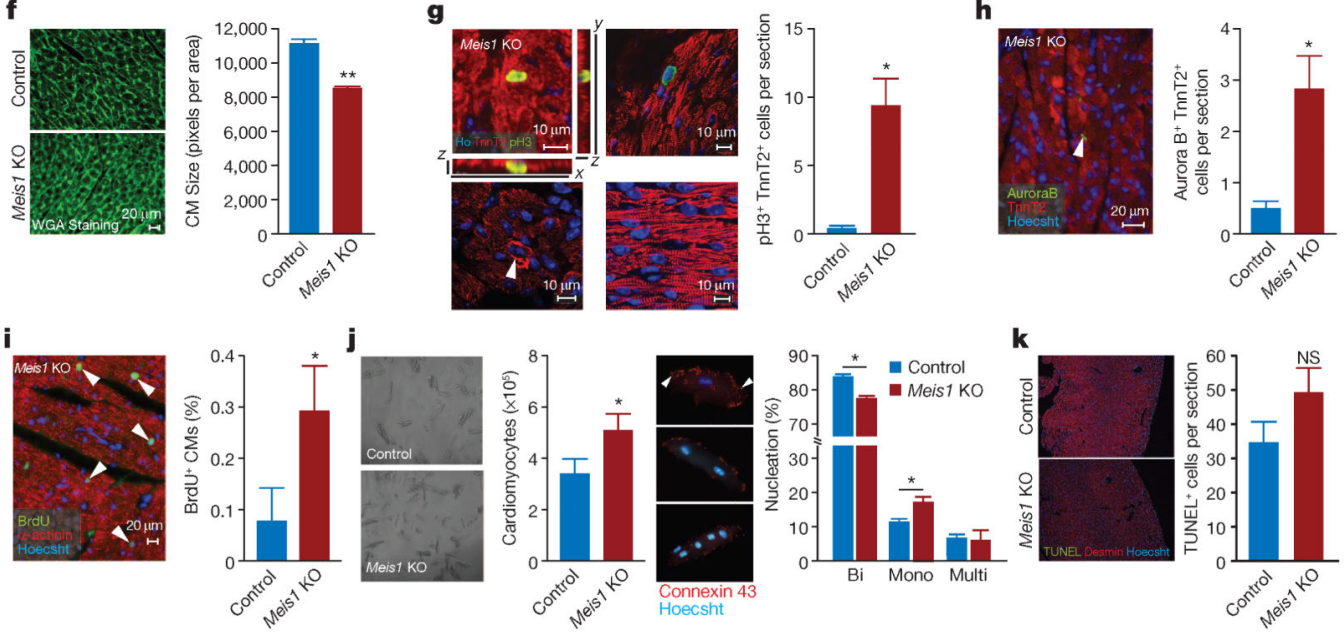

Figure 2. Cardiomyocyte proliferation at P14 following Meis 1 deletion

a, Schematic of Meis1 floxed allele. Control mice were aMHC-Cre, Meis1 KO mice were Meis $1^{\mathrm{f} / \mathrm{f}}$ aMHC-Cre. b, qRT-PCR demonstrates deletion of Meisl in isolated cardiomyocytes at P14 $(n=3)$. c, Trichrome staining of wild-type and Meis1 KO hearts at P14. d, Heart weight (HW) to body weight (BW) ratio in wild-type and Meisl KO hearts ( $n$ = 4-7 per group). e, Left ventricular systolic function quantified by ejection fraction and fractional shortening ( $n=4-7$ per group). f, Wheat germ agglutinin (WGA) staining and cell size quantification. Quantitative analyses represent counting of multiple fields from three independent samples per group ( $\sim 50$ cells per field assessed, total $\sim 250$ cells per group). $\mathbf{g}$, Confocal image with $z$-stacking showing co-localization of $\mathrm{pH} 3$, TnnT2 and Hoechst in a Meis $1 \mathrm{KO}$ heart at P14 (top left). Confocal image of a $\mathrm{pH} 3^{+}$cardiomyocyte in a Meis $1 \mathrm{KO}$ heart (top right). Immunostaining showing sarcomere disassembly in Meis 1 KO hearts (arrowhead, bottom left). Normal sarcomeric structure of Meis1 KO myocardium (bottom right). Graph shows quantification of the number of $\mathrm{pH} 3^{+} \mathrm{TnnT}^{+}$nuclei. $\mathbf{h}$, Immunostaining showing expression of Aurora B in Meis 1 KO cardiomyocytes at P14 and quantification of the number of Aurora $\mathrm{B}^{+} \mathrm{TnnT}^{+}$cardiomyocytes. Quantitative analysis of $\mathrm{pH} 3$ and Aurora $\mathrm{B}^{+}$cardiomyocytes represents counting of multiple sections from three independent samples per group ( 3 sections per heart) (g, h). i, Immunostaining showing colocalization of BrdU, a-actinin and Hoechst in Meis1 KO heart at P14 and quantification of the number of $\mathrm{BrdU}^{+}$cardiomyocytes. Quantification represents counting of several sections from (3-6) independent samples per group. Total number of cardiomyocytes counted for proliferation indices was $2 \times 10^{3}-2.5 \times 10^{3}$ myocytes per section. $\mathbf{j}$, Representative images of control and Meis $1 \mathrm{KO}$ isolated cardiomyocytes, and quantification of the number of myocytes from control and Meis 1 KO hearts. Approximately $1 \times 10^{3}-1.5$ 
$\times 10^{3}$ cardiomyocytes were counted using a haemocytometer per group, using 3 independent samples (left). Immunostaining of isolated cardiomyocytes with Connexin 43, and quantification of the number of nuclei in control and Meisl KO hearts. For nucleation, approximately $1 \times 10^{3}$ cardiomyocytes were counted per sample, using 3 independent samples per group (right). $\mathbf{k}$, Apoptosis analysis. Image showing co-localization of TUNEL, Desmin, and Hoechst in a control and Meisl KO heart. Quantification of TUNEL-positive cells. $(n=3)$. Values presented as mean \pm s.e.m., ${ }^{*} P, 0.05, * * P<0.01$. 

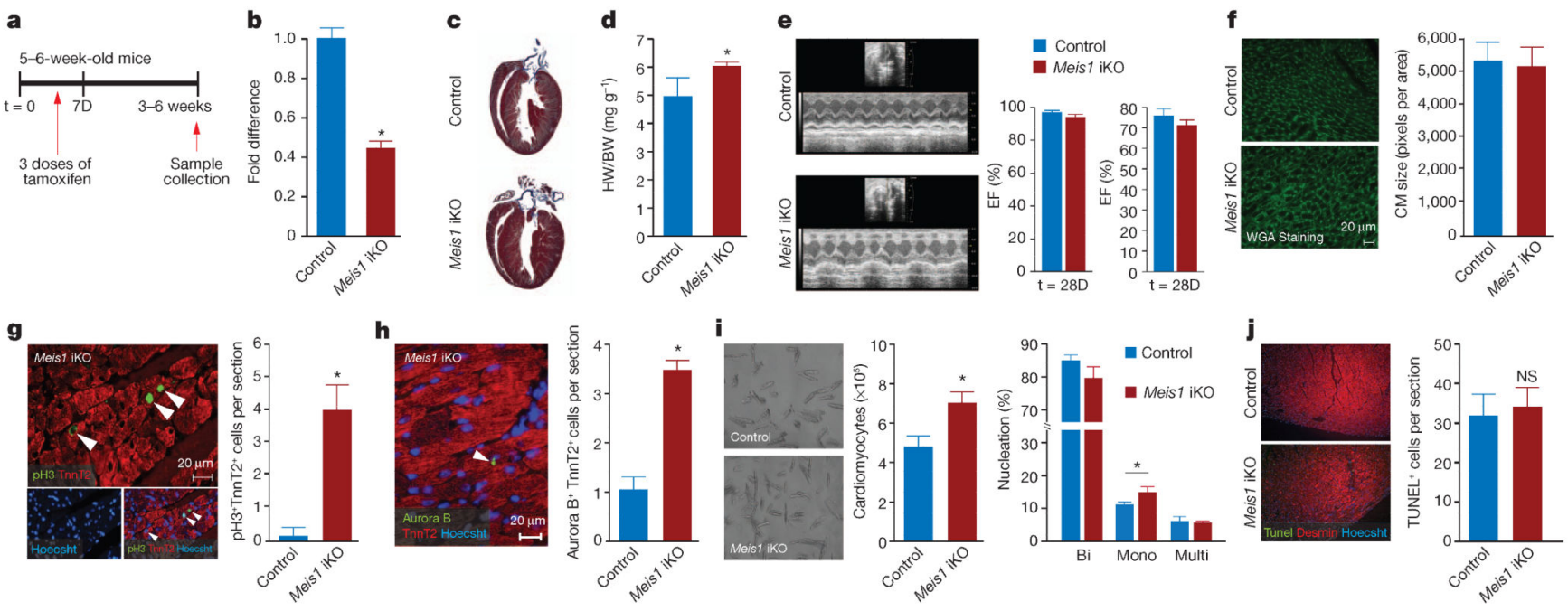

Figure 3. Inducible deletion of Meis1 in cardiomyocytes

a, Schematic of inducible Meis1 deletion (Meis 1 iKO). Control mice were aMHC-Cre MerCreMer, Meisl iKO mice were aMHC-Cre MerCreMer Meis $1^{\mathrm{f} / \mathrm{f}}$. b, qRT-PCR demonstrating deletion of Meisl in isolated cardiomyocytes $(n=3)$. $\mathbf{c}$, Trichrome stained sections at day 28 following tamoxifen injections. d, Heart weight to body weight ratio in wild-type and Meisl iKO mice ( $n=4$ per group). e, Left ventricular systolic function quantified by ejection fraction and fractional shortening ( $n=4$ per group). f, Wheat germ agglutinin staining (WGA) and cell size quantification. Quantitative analyses represent counting of multiple fields from three independent samples per group ( $\sim 50$ cells per field assessed, total $\sim 250$ cells per group). g, Immunostaining image for $\mathrm{pH} 3$, TnnT2 and Hoechst, 7 days post tamoxifen induction. Arrowheads mark $\mathrm{pH} 3^{+}$myocyte nuclei. Graph shows the number of $\mathrm{pH}^{+}{ }^{+} \mathrm{TnnT}_{2}{ }^{+}$nuclei 7 days post tamoxifen treatment in control and Meis 1 iKO hearts $(n=6)$. $\mathbf{h}$, Immunostaining for Aurora B in Meisl iKO heart. Graph shows the number of Aurora $\mathrm{B}^{+}$cardiomyocytes. Quantitative analysis of $\mathrm{pH}^{+}$and Aurora $\mathrm{B}^{+}$myocytes represents counting of multiple sections from three independent samples per group ( $<3$ sections per heart). i, Representative images of control and Meisl iKO isolated cardiomyocytes and quantification of the number of cardiomyocytes. Approximately $1 \times 10^{3}$ $-1.5 \times 10^{3}$ cardiomyocytes were counted using a haemocytometer per group, using 3 independent samples. Graph shows quantification of the nucleation of cardiomyocyte nuclei. For nucleation, approximately $1 \times 10^{3}$ cardiomyocytes were counted per sample, using $\times$ independent samples per group. $\mathbf{j}$, Apoptosis following inducible Meis 1 deletion in cardiomyocytes. Immunostaining for TUNEL, Desmin, and Hoechst in control and Meis 1 iKO hearts and quantification of TUNEL positive cells. Values presented as mean \pm s.e.m., $* P<0.05$. 

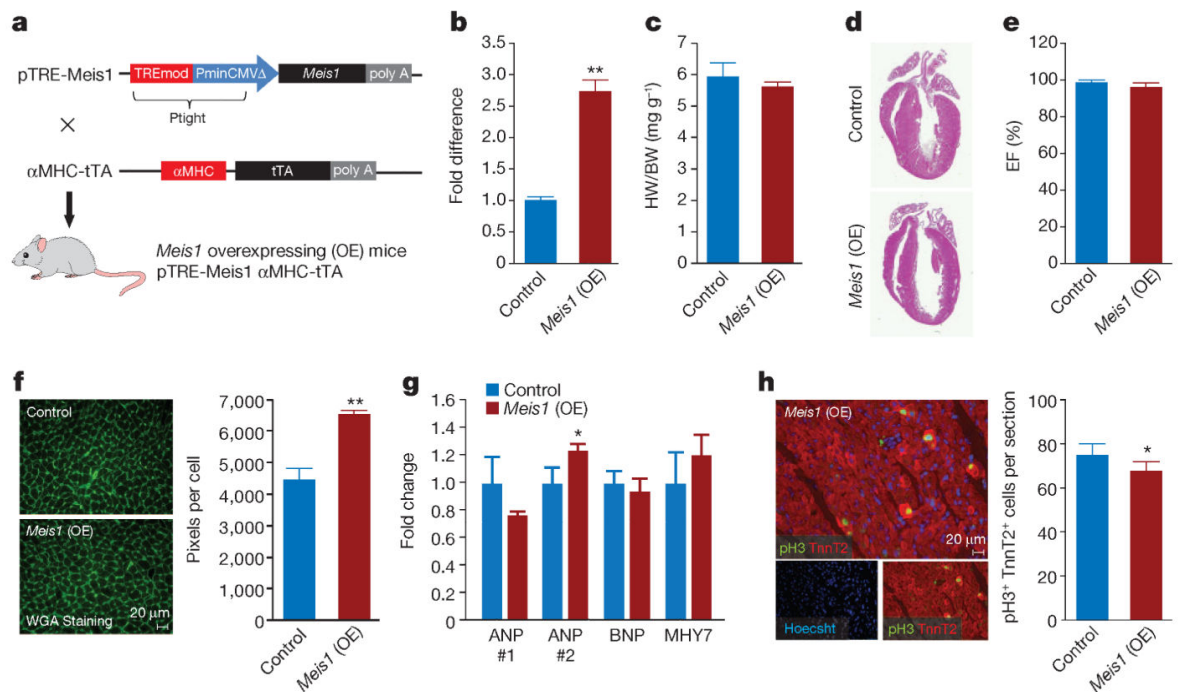

h
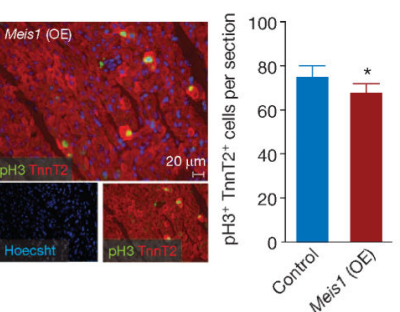

i

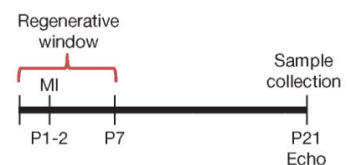

j

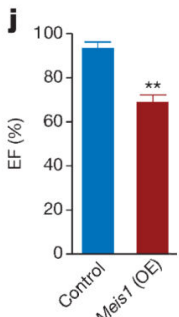

$\mathbf{k}$

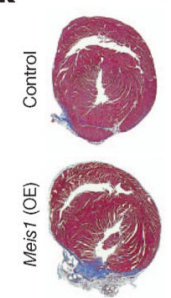

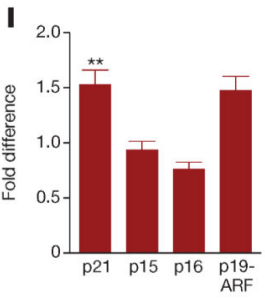

Figure 4. Meis1 overexpression in the heart limits neonatal heart regeneration following myocardial infarction

a, Schematic of Meis1 overexpression (OE) in the heart. Control mice were aMHC-tTA, Meis1 (OE) mice were pTRE-Meis1 aMHC-tTA. b, qRT-PCR demonstrates overexpression of Meisl $(n=3)$. c, Heart weight to body weight ratio in control and Meisl (OE) mice ( $n=$ 11). d, Haematoxylin and eosin stained sections of wild-type and Meisl (OE) hearts. e, Left ventricular systolic function quantified by ejection fraction ( $n=4-5$ per group). $\mathbf{f}$, Wheat germ agglutinin staining and cell size quantification. Quantitative analyses represent counting of multiple fields from three independent samples per group ( $\sim 50$ cells per field assessed, total $\sim 250$ cells per group). g, Gene expression of hypertrophy markers in P3 control and Meis1 (OE) hearts. ANP \#1 and \#2 represent two different primer sets for the same gene. h, Immunostaining image showing co-localization of pH3, TnnT2 and Hoechst in Meisl (OE) heart at P3. Graph shows quantification of the number of $\mathrm{pH}^{+}{ }^{+} \mathrm{TnnT}^{+}$ nuclei. Quantitative analysis represents counting of multiple sections from three independent samples per group ( $\sim 3$ sections per heart). i, Schematic of neonatal MI during the regenerative window at $\mathrm{P} 1 . \mathbf{j}$, Left ventricular systolic function of wild-type and Meis 1 (OE) hearts at 21 days post-MI. k, Masson's trichrome staining at day 21 post-MI. l, qRT-PCR of cyclin dependent kinase inhibitors (CDKIs) in hearts of Meisl (OE) compared to control. Values presented as mean \pm s.e.m; $* P<0.05$, $* * P<0.01$. 